DOI: 10.12731/2227-930X-2020-3-83-98 УДК 629.331

\title{
МАТЕМАТИЧЕСКАЯ МОДЕЛЬ ПРОЦЕССА ДВИЖЕНИЯ АВТОМОБИЛЯ ПО ПОПЕРЕЧНОМУ УКЛОНУ
}

Тихов-Тинников Д.А., Федотов А.И., Алексеев А.В.

В статье представлено аналитическое исследование влияния технического состояния системы подрессоривания на параметры устойчивости автомобиля. Исследуется процесс движения по поперечному уклону при возмущенном состоянии подвески. В качестве оченочных параметров устойчивости движения предложено использовать величины бокового смещения, угла поворота и времени переходного процесса угловой скорости.

Цель - Теоретическое обоснование метода диагностирования подвески по кинематическим параметрам автомобиля.

Методы проведения работы: использованы методы математического моделирования и численные методы решения дифференциальных уравнений.

Результаты: разработан математический аппарат для аналитического исследования процесса движения автомобиля при изменении параметров технического состояния амортизаторов.

Область применения результатов: результаты могут быть использованы организачиями и учреждениями, занимающихся разработкой методов диагностирования автотранспортных средств.

Ключевые слова: автомобиль; амортизатор; подвеска; математическая модель.

\section{MATHEMATICAL MODEL OF THE VEHICLE MOVEMENT PROCESS ON A LATERAL INCLINE}

\author{
Tikhov-Tinnikov D.A., Fedotov A.I., Alekseev A.V.
}

The article presents an analytical study of the influence of the technical state of the suspension system on the stability parameters of the vehicle. 
The process of movement along the transverse slope with the disturbed state of the suspension is investigated. It is proposed to use the values of the lateral displacement, the angle of rotation and the time of the transient process of the angular velocity as the estimated parameters of motion stability.

Purpose - Theoretical substantiation of the method for diagnosing the suspension by the kinematic parameters of the vehicle.

Methodology includes methods of mathematical modeling and numerical methods for solving differential equations.

Results: a mathematical apparatus was developed for the analytical study of the process of vehicle movement when changing the parameters of the technical state of shock absorbers.

Practical implications: the results can be used by organizations and institutions involved in the development of diagnostic methods for vehicles.

Keywords: car; shock absorber; suspension; mathematical model.

\section{Введение}

В настоящее время исследование влияния технического состояния подвески на устойчивость автотранспортных средств (АТС) в условиях эксплуатации является актуальной научно-технической задачей [1-3], для решения которой используются теоретические [46] и экспериментальные методы научных исследований [7-12]. Для условий эксплуатации предлагается использовать дорожный метод контроля технического состояния подвески по критериям устойчивости при движении автомобиля по поперечному уклону и возмущённом состоянии подвески [13]. Проведение дорожного эксперимента требует тщательного планирования и подготовки. В связи с этим на текущем этапе работы исследование проводится аналитически с использованием методов математического моделирования. Для выполнения теоретических исследований необходимо разработать математическое описание исследуемых процессов в соответствии с нижеследующей постановкой задачи. АТС движется по ровному участку дороги с поперечным уклоном $\varepsilon$. На поверхности дороги расположена выступающая единичная неровность прямоугольной формы высотой $h_{i r}$ и длиной $l_{i r}$. До момента наезда передни- 
ми колесами на неровность за счёт рулевого управления обеспечивается траектория движения параллельная продольной оси дороги. Далее углы поворота управляемых колёс $\Theta_{11}$ и $\Theta_{12}$ остаются неизменными. В начальный момент времени скорость АТС равна нулю, затем скорость увеличивается до заданного уровня, который сохраняется до конца испытания. Требуется определить влияние технического состояния амортизаторов на траекторию движения АТС после взаимодействия колёс с единичной неровностью. Результатом аналитической работы должно стать теоретическое обоснование возможности выполнения оценки влияния технического состояния подвески на устойчивость движения АТС в условиях эксплуатации.

\section{Методы и материалы}

Для решения поставленной задачи разработана расчётная схема (рис.1). Движение автомобиля рассматривается в двух системах координат - подвижной и неподвижной.

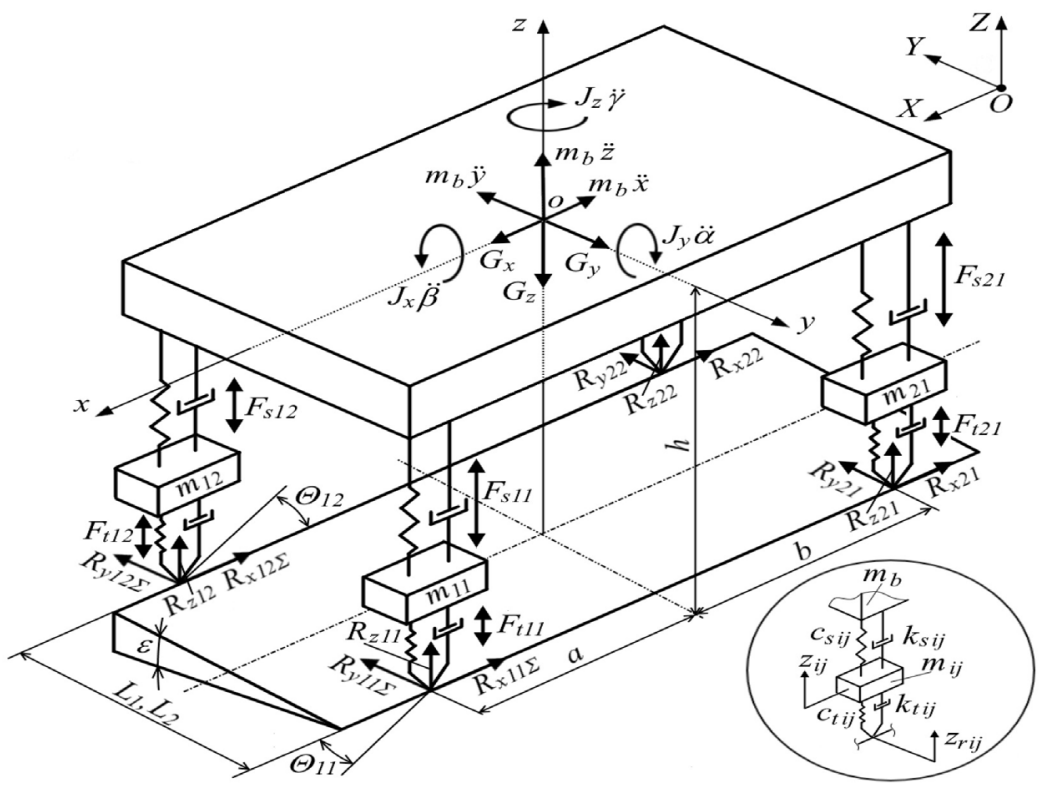

Рис. 1. Расчетная схема процесса движения АТС по поперечному уклону 
Центр подвижной системы $x y z$ постоянно связан с центром масс автомобиля. Ось $о x$ совпадает с продольной осью автомобиля, ось оу с поперечной. Оси неподвижной системы координат $X Y Z$ параллельны горизонтальным и вертикальной осям пространства. Нулевые значения неподвижных координат определяются начальным положением центра масс автомобиля в момент времени $t=0$.

Для описания движения автомобиля рассмотрим систему, состоящую из четырёх неподрессоренных масс, взаимодействующих с опорной поверхностью и неподрессоренной массой посредством упругих и демпфирующих связей. В модели динамика подрессоренной массы рассчитывается по принципу Даламбера по шести степеням свободы (1), а динамика неподрессоренных масс - только по вертикальной оси (2). Системы уравнений (1) и (2) являются нелинейными и для решения используем метод численного интегрирования Эйлера:

$$
\begin{aligned}
& \left\{\begin{array}{c}
m_{b}(\ddot{x}+\dot{\alpha} \dot{z}-\dot{\gamma} \dot{y})=\Sigma F_{x} \\
m_{b}(\ddot{y}+\dot{\gamma} \dot{x}-\dot{\beta} \dot{z})=\Sigma F_{y} \\
m_{b}(\ddot{z}+\dot{\beta} \dot{y}-\dot{\alpha} \dot{x})=\Sigma F_{z} \\
J_{X} \ddot{\beta}+\left(J_{Z}-J_{Y}\right) \dot{\gamma} \dot{\alpha}=\Sigma M_{x} \\
J_{Y} \ddot{\alpha}+\left(J_{X}-J_{Z}\right) \dot{\beta} \dot{\gamma}=\Sigma M_{y} \\
J_{Z} \ddot{\gamma}+\left(J_{Y}-J_{X}\right) \dot{\alpha} \dot{\beta}=\Sigma M_{Z}
\end{array}\right. \\
& \left\{m_{i j} \ddot{z}_{i j}=-m_{i j} g+F_{s i j}+F_{t i j}\right. \text {. }
\end{aligned}
$$

где: $m_{b}$ - масса подрессоренной части; $\dot{x}, \dot{y}, \dot{z}$ и $\ddot{x}, \ddot{y}, \ddot{z}-$ проекции векторов скорости и ускорения подрессоренной массы на подвижную систему координат; $\dot{\alpha}, \dot{\beta}, \dot{\gamma}$ и $\ddot{\alpha}, \ddot{\beta}, \ddot{\gamma}-$ угловые скорости и ускорения подрессоренной массы; $J_{X} J_{Y} J_{Z}$ - осевые моменты инерции кузова АТС; $\Sigma F_{x}, \Sigma F_{y}, \Sigma F_{z}-$ суммы проекций сил и реакций на оси подвижной системы координат, определяемые по выражениям (3$5) ; \Sigma M_{x}, \Sigma M_{y}, \Sigma M_{z}$ - суммы крутящих моментов от сил и реакций относительно осей подвижной системы координат (6-8); $m_{i j}$ - масса неподрессоренной части (здесь и далее $i=1-$ передняя ось, $i=2-$ задняя ось, $j=1-$ левое колесо, $j=2-$ правое колесо); $g$ - ускорение свободного падения; $F_{s i j}-$ усилия взаимодействия подрессоренной 
и неподрессоренной массы (9); $F_{t i j}$ - усилия взаимодействия неподрессоренной массы с опорной поверхностью дороги (10).

$$
\begin{aligned}
& \Sigma F_{x}=G_{x}-R_{x 11 \Sigma}-R_{x 12 \Sigma}-R_{x 21}-R_{x 22}, \\
& \Sigma F_{y}=-G_{y}-R_{y 11 \Sigma}+R_{y 12 \Sigma}+R_{y 21}+R_{y 22}, \\
& \Sigma F_{z}=-G_{z}+F_{s 11}+F_{s 12}+F_{s 21}+F_{s 22}, \\
& \Sigma M_{x}=0.5\left(\left(F_{s 12}-F_{s 11}\right) L_{1}+\left(F_{s 22}-F_{s 21}\right) L_{2}\right)-\left(R_{y 11 \Sigma}(h-\right. \\
& \left.\left.-z_{r 11}\right)+R_{y 12 \Sigma}\left(h-z_{r 12}\right)+R_{y 21}\left(h-z_{r 21}\right)+R_{y 22}\left(h-z_{r 22}\right)\right), \\
& \Sigma M_{y}=\left(F_{s 11}+F_{s 12}\right) a-\left(F_{s 21}-F_{s 22}\right) b-R_{x 11 \Sigma}\left(h-z_{r 11}\right)- \\
& -R_{x 12 \Sigma}\left(h-z_{r 12}\right)-R_{x 21}\left(h-z_{r 21}\right)-R_{x 22}\left(h-z_{r 22}\right), \\
& \Sigma M_{z}=\left(R_{y 11 \Sigma}+R_{y 12 \Sigma}\right) a-\left(R_{y 21}+R_{y 22}\right) b+0.5\left(\left(R_{x 11 \Sigma}-\right.\right. \\
& \left.\left.-R_{x 12 \Sigma}\right) L_{1}+\left(R_{x 21}-R_{x 22}\right) L_{2}\right), \\
& F_{s i j}=c_{s i j}\left(z_{i j}-z_{s i j}\right)+k_{s i j}\left(\dot{z}_{i j}-\dot{z}_{s i j}\right), \\
& F_{t i j}=c_{t i j}\left(z_{r i j}-z_{i j}\right)+k_{t i j}\left(\dot{z}_{r i j}-\dot{z}_{i j}\right) .
\end{aligned}
$$

В уравнениях (3-10): $G_{x}, G_{y}$ и $G_{z}-$ - проекции веса подрессоренной части на оси подвижной системы координат (11 - 13); $R_{x 1 ; \Sigma}$ и $R_{y 1 j \Sigma}-$ суммы проекций продольных и поперечных реакций передних колес на оси ох и оу соответственно $(14,15) ; R_{x i j}$ и $R_{y i j}-$ продольные и поперечные реакции колес; $L_{1}$ и $L_{2}-$ колеи передних и задних колес; $h$ - вертикальная координата центра масс (17); $z_{i j}$ и $\dot{Z}_{i j}$ - вертикальная координата и вертикальная скорость неподрессоренных масс; $z_{s i j}$ и $\dot{z}_{s i j}-$ вертикальные координаты (17) и скорости подрессоренной массы над точками контакта колес с опорной поверхностью (18); $z_{r i j}$ и $\dot{z}_{r i j}$ - вертикальная координата и условная вертикальная скорость опорной поверхности по колесам автомобиля; $a$ и $b$ - расстояние от передней и задней осей до центра масс; $c_{s i j}$ - жесткость подвески; $k_{s i j}$ - коэффициент демпфирования амортизатора; $c_{t i j}$ - жесткость шины; $k_{t i j}$ - коэффициент демпфирования шины. 


$$
\begin{aligned}
& G_{x}=m_{b} g \sin (\varepsilon) \sin (\gamma) \\
& G_{y}=m_{b} g \sin (\varepsilon) \cos (\gamma), \\
& G_{z}=m_{b} g \cos (\varepsilon) \\
& R_{x 1 j \Sigma}=R_{x 1 j} \cos \theta_{1 j}+R_{y 1 j} \sin \theta_{1 j}, \\
& R_{y 1 j \Sigma}=R_{y 1 j} \cos \theta_{1 j}-R_{x 1 j} \sin \theta_{1 j}, \\
& h=\left(h_{d}-z_{s t}+z\right) \\
& z_{s i j}=z+(3-2 i)(a(2-i)+b(i-1)) \sin \alpha-(1.5-j) L_{j} \sin \beta
\end{aligned}
$$

В уравнениях (11-17): $\gamma$ - накопленный угол поворота автомобиля; $\theta_{1}$ - угол поворота переднего левого колеса; $\theta_{2}$ - угол поворота переднего правого колеса; $\alpha$ и $\beta$ - углы поворота подрессоренной массы относительно осей $о x$ и оу соответственно; $h_{d}$ - высота центра масс; $h_{s t}$ - вертикальная координата центра масс при статическом прогибе подвески; $z$ - текущая вертикальная координата центра масс.

Продольные и поперечные реакции $R_{x i j}$ и $R_{y i j}$ в пятне контакта рассчитываются на основе нормированной функции проскальзывания по методике [14] .

Единичная неровность задается в виде функции, описывающей сглаживающую способность шины [15].

Скорость бокового смещения $V_{Y}$ относительно неподвижной системы координат определяется уравнением

$$
V_{Y}=\dot{x}_{b} \sin \gamma+\dot{y}_{b} \cos \gamma
$$

Прямолинейное движение АТС до момента наезда на неровность определяется обеспечивается изменениям углов поворота колес передней оси $\theta_{1}$ и $\theta_{2}$. В зависимости от текущего положения АТС каждый из углов может являться как внутренним $\theta_{\text {int }}$ так и наружным $\theta_{\text {out }}$ по отношению траектории движения. Коррекция расчетной траектории производится по углу поворота внутреннего колеса

$$
\theta_{\text {int }}=\theta_{\text {int }}^{*}-k_{\theta} V_{Y}
$$

где: $\theta_{i n t}^{*}-$ значение угла поворота внутреннего колеса на предыдущем шаге интегрирования, $k_{\theta}$ - параметр, определяющий зависимость приращения угла поворота внутреннего колеса от скорости бокового смещения $V_{Y}$, имеющий размерность сек/м. 
Из известного соотношения углов поворота управляемых колес определяется значение угла поворота внешнего колеса

$$
\theta_{\text {out }}=\operatorname{arcctg}\left(\operatorname{ctg} \theta_{\text {int }}+L_{1} /(a+b)\right) .
$$

Определение принадлежности углов $\theta_{1}$ и $\theta_{2}$ к внутреннему и внешнему колесу осуществляется с использованием системы логических выражений

$$
\left\{\begin{array}{c}
V_{Y}=0 \Rightarrow \theta_{1}=0 \Rightarrow \theta_{2}=0 \\
V_{Y} \neq 0 \wedge \theta_{1} \geq 0 \Rightarrow \theta_{1}=\theta_{\text {int }} \Rightarrow \theta_{2}=\theta_{\text {out }} \\
V_{Y} \neq 0 \wedge \theta_{1}<0 \Rightarrow \theta_{1}=\theta_{\text {out }} \Rightarrow \theta_{2}=\theta_{\text {int }}
\end{array}\right.
$$

Выражения (1-21) составляют основу разработанной компьютерной программы [16] для расчета параметров движения АТС по уклону с переездом единичной неровности. При выполнении расчетов использовался пакет прикладных математических программ Scilab [17].

\section{Результаты}

Используя разработанное математическое описание моделировалось движение механического транспортного средства категории M1, параметры которого аналогичными, описанным в источнике [18]. Высота единичной неровности $h_{i r}=0,05 \mathrm{~m}$, длина $l_{i r}=$ 0,05 м. Разгон АТС осуществлялся до скорости 10 м/с. В результате получены траектории движения АТС при различных углах поперечного уклона $\varepsilon$ от $5^{0}$ до $35^{\circ}$ с шагом $5^{0}$ (рис. 1).

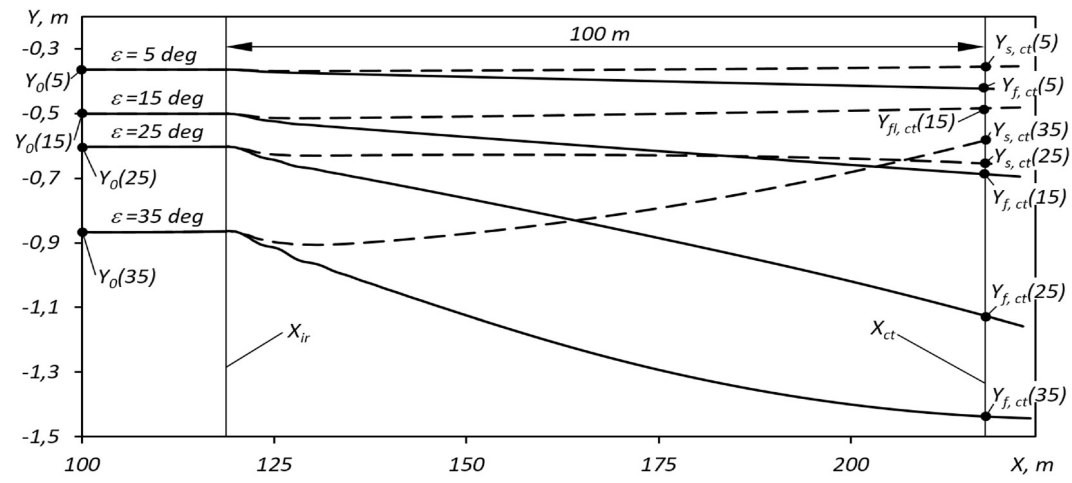

Рис. 1. Расчетные траектории движения АТС при различных углах поперечного уклона $\varepsilon$ 
Пунктирными линиями на рис. 1 показаны траектории движения автомобиля с исправной подвеской, сплошными - с четырьмя неисправными амортизаторами. Усилия, развиваемые неисправными амортизаторами, составляли $20 \%$ от нормативного значения. Линия $\mathrm{X}_{i r}$ обозначает абсциссу расположения в системе неподвижных координат продольной оси единичной неровности. Линия $\mathrm{X}_{c t}$ соответствует прохождению АТС контрольного участка длиной $100 \mathrm{M.}$

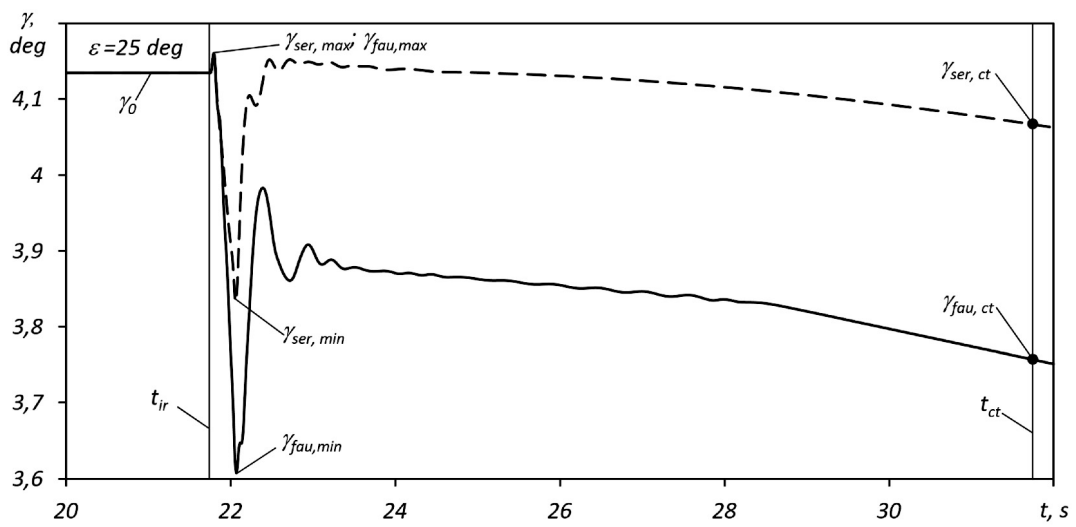

Рис. 2. Расчетная характеристика изменения угла поворота $\gamma$ от времени при движении АТС по опорной поверхности с поперечным уклоном

Для каждой траектории также были рассчитаны зависимости изменения угла поворота $\gamma$ от времени. На рис. 2 представлены характеристики, полученные при моделировании движения АТС по поперечному уклону при $\varepsilon=25^{0}$. Пунктирная линия соответствует исправной подвеске, сплошная - неисправной.

По аналогии с графиками траекторий движения, здесь $t_{i r}$ обозначает время наезда передних колес на единичную неровность, $t_{c t}$ - время проезда контрольного участка.

\section{Обсуждение и заключение}

Анализ расчетных траекторий движения (рис. 1) позволяет охарактеризовать некоторые свойства разработанной математической 
модели. В частности, при движении по поперечному уклону до наезда на единичную неровность прямолинейная траектория находится на некотором смещении от нулевой ординаты горизонтальной плоскости. Чем больше $\varepsilon$ тем сильнее смещение. Указанную особенность необходимо учитывать при выполнении совместного анализа траекторий движения АТС, полученных при разных значениях величины бокового уклона $\varepsilon$. Потребуется дополнительная математическая обработка результатов расчета в целях приведения ординат траекторий $Y_{0}(\varepsilon)$ к одному уровню в момент наезда АТС на единичную неровность.

Моделирование движения на контрольном участке показывает, что при возрастании величины поперечного уклона повышается чувствительность модели к изменению технического состояния подвески, т.е. наблюдается увеличение расстояния между контрольными точками $Y_{s, c t}(\varepsilon)$ и $Y_{f, c t}(\varepsilon)$ для исправного и неисправного состояний подвески соответственно. Однако, при $\varepsilon>25^{0}$ расчетная траектория АТС с исправной подвеской претерпевает качественные изменения по сравнению с траекториями меньших уклонов. Направление движение меняется на обратное и АТС двигается вверх по уклону. Данное явление объясняется применением в модели упрощенного математического аппарата (21) для регулирования прямолинейного движения, который не обеспечивает стабилизацию углов поворота управляемых колес на одном уровне до момента наезда на неровность. В связи с этим область определения модели по углу поперечного уклона ограничивается диапазоном от $0^{0}$ до $25^{\circ}$.

Модель также показывает наличие чувствительности угла поворота $\gamma$ к изменению технического состояния подвески (рис. 2). При этом о состоянии подвески можно судить как по разности накопленных значений углов $\gamma_{s e r, c t}-\gamma_{f a u, c t}$, рассчитанных в конце проезда контрольного участка, так и по разности минимальных значений $\gamma_{s e r, m i n}-\gamma_{f a u, m i n}$, определяемых после проезда АТС единичной неровности.

В классической теории автомобиля [19] оценку устойчивости движения АТС дают по характеру изменения первой производной 
угла поворота $\omega=d \gamma / d t$. На рис. 3 представлены зависимости изменения угловой скорости от времени для АТС с исправной и с неисправной подвеской.

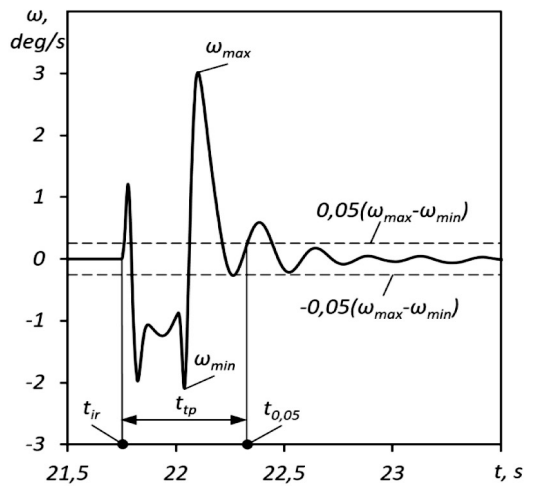

a)

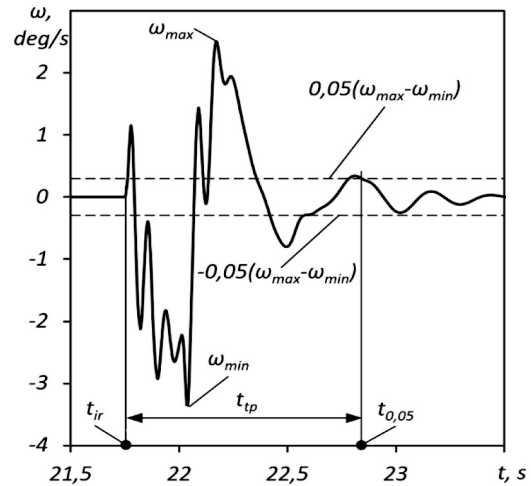

b)

Рис. 3. Изменение угловой скорости АТС $\omega$ (расчет на модели) после переезда единичной неровности при движении по поперечному уклону $\varepsilon=25^{\circ}$. Подвеска: a) - исправная, $b$ ) - неисправная.

Оценочным параметром в этом случае является время протекания переходного процесса, определяемое моментом стабилизации угловой скорости в диапазоне $\pm 5 \%$ от величины максимальной амплитуды колебания $\omega_{\max }-\omega_{\min }$. Расчеты показывают наличие влияния параметров технического состояния подвески на время переходного процесса. Снижение усилия демпфирования амортизаторов до $20 \%$ от номинального значения увеличивает время переходного процесса $t_{t p}$ на $56,5 \%$.

Таким образом, разработанная математическая модель процесса движения автомобиля по поперечному уклону с переездом единичной неровности свидетельствует о теоретической возможности выполнения в условиях эксплуатации оценки влияния технического состояния подвески на устойчивость движения АТС. Критериями оценки могут быть параметры, измеряемые при движении автомобиля после проезда единичной неровности: боковое смещение, угол поворота и время переходного процесса угловой скорости. 
Информация о конфликте интересов. Авторы заявляют об отсутствии конфликта интересов.

Информация о спонсорстве. Исследование не имело спонсорской поддержки.

\section{Сиисок литературы}

1. Федотов А.И., Лысенко А.В., Тихов-Тинников Д.А. Контроль технического состояния подвески автомобилей в условиях эксплуатации методом движения по окружности // Автомобиль для Сибири и Крайнего Севера. Конструкция, эксплуатация, экономика: материалы 90-й Международной научно-технической конференции. Иркутск: ИРНИТУ, 2015. С. 232-237.

2. Кузнецов Н.Ю. Контроль технического состояния автомобильных амортизаторов на основе характеристик сцепления шин с опорной поверхностью: дис. ... канд. тех. наук. Иркутск, 2019. 221 с.

3. Лысенко А.В. Дорожный метод контроля технического состояния амортизаторов в условиях эксплуатации: дис. ... канд. тех. наук. Иркутск, 2019. 293 с.

4. Динамика системы дорога - шина - автомобиль - водитель / Хачатуров А.А., Афанасьев В.Л., Васильев В.С., Гольдин Г.В., Додонов Б.М., Жигарев В.П., Кольцов В.И., Юрик В.С., Яковлев Е.И. Под ред. Хачатурова А.А. М: Машиностроение, 1976. 535 с.

5. Малюгин П.Н., Немчинов Д.В. Торможение колеса на косогоре // Сборник материалов II Национальной научно-практической конференции «Образование. Транспорт. Инновации. Строительство». Омск: СибАДИ, 2019. С. 290-293.

6. Simniceanu L. The Study of the Car's Stability Using a Simplified Model // IOP Conference Series: Materials Science and Engineering. 2019. №568(1). doi:10.1088/1757-899x/568/1/012053

7. Кузнецов Н.Ю., Лысенко А.В., Зедгенизов В.Г. Экспериментальное исследование процесса переезда автомобилем единичной неровности // Вестник Иркутского государственного технического университета. 2017. Т. 21. № 11 (130). С. 191-198.

8. Кичигин Д.В., Зарщиков А.М. Стенд для испытания автомобильных шин в тормозном режиме // Сборник материалов II Наци- 
ональной научно-практической конференции «Образование. Транспорт. Инновации. Строительство». Омск: СибАДИ, 2019. C. 111-114.

9. Назарько О.А. Экспериментальное исследование устойчивости легкового автомобиля в тяговом режиме движения с помощью датчиков линейного ускорения // Ученые записки Крымского инженерно-педагогического университета. 2012. № 36. С. 26-29.

10. Prochowski L., Pusty T., Gidlewski M., Jemioł L. Experimental studies of the car-trailer system when passing by a suddenly appearing obstacle in the aspect of active safety of autonomous vehicles // IOP Conference Series: Materials Science and Engineering. 2018. №421(3). doi:10.1088/1757-899x/421/3/032024

11. Heerwan P.M., Asyraf S.M., Efistein A.N., Seah C.H., Zikri J.M., Syawahieda, J.N. Experimental study of the vehicle dynamics behavior during lane changing in different speeds // IOP Conference Series: Materials Science and Engineering. 2017. №257(1). doi:10.1088/1757899x/257/1/012078.

12. Abdullah M.A., Jamil J.F., Salim M.A. Dynamic performances analysis of a real vehicle driving // IOP Conference Series: Materials Science and Engineering. 2015. №100(1). doi: 10.1088/1757-899X/100/1/012017

13. Федотов А.И., Лысенко А.В., Тихов-Тинников Д.А., Контроль технического состояния подвески автомобилей в условиях эксплуатации методом движения по поперечному уклону // Журнал автомобильных инженеров. 2015. № 6. С. 51-53.

14. Дик А.Б. Расчет стационарных и нестационарных характеристик тормозящего колеса при движении с уводом: дис. ... канд. тех. наук. Омск, 1988. 228 с.

15. Неволин Д.Г., Новосёлов Л.И. Математическое моделирование динамического процесса в подвески полуприцепа // Транспорт Урала. 2014. №4. С. 119-122

16. Свидетельство о государственной регистрации программы для ЭВМ №2020660484. Расчет параметров процесса движения автомобиля по уклону с переездом единичной неровности / Тихов-Тинников Д.А., Федотов А.И. - Заявка №2020619758. Дата 
поступления 02 сентября 2020 г. Зарегистрировано в Реестре программ для ЭВМ 04 сентября 2020 г.

17. Scilab URL: https://www.scilab.org/ (дата обращения: 13.10.2020).

18. Fedotov A.I., Tikhov-Tinnikov D.A., Ovchinnikova N.I., Lysenko A.V. Simulation of car movement along circular path // IOP Conference Series: Earth and Environmental Science. 2018. №87(8). doi:10.1088/1755-1315/87/8/082018

19. Литвинов А.С., Фаробин Я.Е. Автомобиль: Теория эксплуатационных свойств: Учебник для вузов по специальности «Автомобили и автомобильное хозяйство». М.: Машиностроение, 1989. 240 с.

\section{References}

1. Fedotov A.I., Lysenko A.V., Tikhov-Tinnikov D.A. Materialy 90-j Mezhdunarodnoj nauchno-tehnicheskoj konferencii «Avtomobil'dlja Sibiri i Krajnego Severa. Konstrukcija, jekspluatacija, jekonomika” [Materials of the 90th International Scientific and Technical Conference "Car for Siberia and the Far North. Design, operation, economy"]. Irkutsk, 2015. pp. 232-237.

2. Kuznecov N.Ju. Kontrol' tehnicheskogo sostojanija avtomobil'nyh amortizatorov na osnove harakteristik sceplenija shin s opornoj poverhnost'ju [Monitoring the technical condition of automobile shock absorbers based on tire grip characteristics]. Irkutsk: dis. ... cand. tech. sci., 2019. 221 p.

3. Lysenko A.V. Dorozhnyj metod kontrolja tehnicheskogo sostojanija amortizatorov $v$ uslovijah jekspluatacii [Road method for monitoring the technical condition of shock absorbers under operating conditions]. Irkutsk: dis. ... cand. tech. sci., 2019. 293 p.

4. Hachaturov A.A., Afanas'ev V.L., Vasil'ev V.S., Gol'din G.V., Dodonov B.M., Zhigarev V.P., Kol'cov V.I., Jurik V.S., Jakovlev E.I. Dinamika sistemy doroga - shina - avtomobil' - voditel' [Dynamics of a system road - tyre - car-driver]. Moscow: Mashinostroenie, 1976. 535 p.

5. Maljugin P.N., Nemchinov D.V. Sbornik materialov II Nacional'noj nauchno-prakticheskoj konferencii "Obrazovanie. Transport. Innovacii. Stroitel'stvo"[Compilation of materials of the II National Sci- 
entific and Practical Conference "Education. Transport. Innovations. Construction"]. Omsk, 2019. pp. 290-293.

6. Simniceanu L. IOP Conference Series: Materials Science and Engineering, 568 (2019). doi:10.1088/1757-899x/568/1/012053

7. Kuznecov N.Ju., Lysenko A.V., Zedgenizov V.G. Vestnik Irkutskogo gosudarstvennogo tehnicheskogo universiteta, no.11 (2017): 191-198.

8. Kichigin D.V., Zarshhikov A.M. "Obrazovanie. Transport. Innovacii. Stroitel'stvo" [Compilation of materials of the II National Scientific and Practical Conference "Education. Transport. Innovations. Construction"]. Omsk, 2019. pp. 111-114.

9. Nazar'ko O. A. Uchenye zapiski Krymskogo inzhenerno-pedagogicheskogo universiteta, no.36 (2012): 26-29.

10. Prochowski L., Pusty T., Gidlewski M., Jemioł L. Experimental studies of the car-trailer system when passing by a suddenly appearing obstacle in the aspect of active safety of autonomous vehicles. IOP Conference Series: Materials Science and Engineering. 2018. №421(3). doi:10.1088/1757-899x/421/3/032024

11. Heerwan P.M., Asyraf S.M., Efistein A.N., Seah C.H., Zikri J.M., Syawahieda, J.N. Experimental study of the vehicle dynamics behavior during lane changing in different speeds. IOP Conference Series: Materials Science and Engineering. 2017. №257(1). doi:10.1088/1757-899x/257/1/012078.

12. Abdullah M.A., Jamil J.F., Salim M.A. Dynamic performances analysis of a real vehicle driving. IOP Conference Series: Materials Science and Engineering. 2015. №100(1). doi: 10.1088/1757-899X/100/1/012017

13. Fedotov A.I., Lysenko A.V., Tikhov-Tinnikov D.A. Zhurnal avtomobil'nyh inzhenerov, no.6 (2015): 51-53.

14. Dik A.B. Raschet stacionarnyh i nestacionarnyh harakteristik tormozjashhego kolesa pri dvizhenii s uvodom [Calculation of Stationary and Non-Stationary Characteristics of the Brake Wheel when Driving with a Slip Angle]. Omsk dis. ... cand. tech. sci., 1988. 228 p.

15. Nevolin D.G., Novoselov L.I. Transport Urala, no.4 (2014): 119-122.

16. Svidetel'stvo o gosudarstvennoj registracii programmy dlja JeVM №2020660484. Raschet parametrov processa dvizhenija avtomobilja po uklonu s pereezdom edinichnoj nerovnosti / Tikhov-Tinnikov D.A., Fedo- 
tov A.I. - Zajavka №2020619758. Data postuplenija 02 sentjabrja 2020 g. Zaregistriro-vano v Reestre programm dlja JeVM 04 sentjabrja $2020 \mathrm{~g}$. [Certificate of state registration of the computer program No. 2020660484. Calculation of the parameters of the process of movement of the car along the slope with the crossing of a single unevenness / Tikhov-Tinnikov D.A., Fedotov A.I. - Application No. 2020619758. Date of receipt September 2, 2020. Registered in the Register of computer programs September 4, 2020.]

17. Scilab. URL: https://www.scilab.org/ (accessed October 10, 2020)

18. Fedotov A.I., Tikhov-Tinnikov D.A., Ovchinnikova N.I., Lysenko A.V. Simulation of car movement along circular path. IOP Conference Series: Earth and Environmental Science. 2018. №87(8). doi:10.1088/1755-1315/87/8/082018

19. Litvinov A.S., Farobin Ya.E. Avtomobil': Teorija jekspluatacionnyh svojstv: Uchebnik dlja vuzov po special'nosti «Avtomobili i avtomobil'noe hozjajstvo» [Automobile: Theory of operational properties: A textbook for universities in the specialty "Automobiles and Automotive Industry”]. M.: Mashinostroenie, 1989.240 p.

\section{ДАННЫЕ ОБ АВТОРАХ}

Тихов-Тинников Дмитрий Анатольевич, старший научный сотрудник управления научных исследований, кандидат технических наук, доцент

Восточно-Сибирский государственный университет технологий и управления

ул. Ключевская, 40В, строение 1, г. Улан-Удэ, Республика Бурятия, 670013, Российская Федеращия

dm_tt@mail.ru

Федотов Александр Иванович, заведующий кафедрой автомобильного транспорта, доктор технических наук, профессор Иркутский нащиональный исследовательский технический университет

ул. Лермонтова, 83, г. Иркутск, Иркутская область, 664074, Российская Федерация

fai.abs@yandex.ru 
Алексеев Алексей Васильевич, доцент кафедры «Автомобили», кандидат технических наук

Восточно-Сибирский государственный университет технологий и управления

ул. Ключевская, 40В, строение 1, г. Улан-Удэ, Республика Бурятия, 670013, Российская Федерация

kafautoalexey@gmail.com

\section{DATA ABOUT THE AUTHORS}

Tikhov-Tinnikov Dmitry Anatolyevich, Senior Researcher at the Research Department, Candidate of Technical Sciences, Associate Professor

East Siberian State University of Technology and Management 40B/1, Klyuchevskaya St., Ulan-Ude, Republic of Buryatia, 670013, Russian Federation

dm tt@mail.ru

ORCID: 0000-0003-0912-4109

Fedotov Alexander Ivanovich, Head of the Department of Road Transport, Doctor of Technical Sciences, Professor

Irkutsk National Research Technical University

83, Lermontov St., Irkutsk, Irkutsk region, 664074, Russian Federation

fai.abs@yandex.ru

Scopus Author ID: 56341065000

Alekseev Alexey Vasilyevich, Associate Professor of the Department of Cars, Candidate of Technical Sciences

East Siberian State University of Technology and Management

40B/1, Klyuchevskaya St., Ulan-Ude, Republic of Buryatia, 670013, Russian Federation

kafautoalexey@gmail.com

ORCID: 0000-0002-4279-0683 